Original Research Paper

\title{
Diversity and Allelopathic Potential of Weeds among Panamanian Coffee Crops
}

\author{
${ }^{1}$ Lilia Cherigo, ${ }^{2}$ Jorge Lezcano and ${ }^{3}$ Sergio Martínez-Luis \\ ${ }^{1}$ Departamento de Química Orgánica, \\ Facultad de Ciencias Exactas y Tecnología, Universidad de Panamá, Panamá \\ ${ }^{2}$ Secretaria de Competitividad y Logística, \\ Ministerio de la Presidencia, Palacio de Las Garzas, Ciudad de Panamá, República de Panamá \\ ${ }^{3}$ Centro de Biodiversidad y Descubrimiento de Drogas, \\ Instituto de Investigación Científica y Servicios de Alta Tecnología, Clayton, Ciudad del Saber, Republica de Panamá
}

Article history

Received: 24-05-2017

Revised: $10-08-2017$

Accepted: 22-08-2017

Corresponding Author:

Sergio Martínez-Lui

Centro de Biodiversidad y

Descubrimiento de Drogas,

Instituto de Investigación

Científica y Servicios de Alta

Tecnología, Clayton, Ciudad

del Saber, Republica de

Panamá

Email: smartinez@indicasat.org.pa

\section{Introduction}

The first coffee plantations in Panama sprung up in the early nineteenth century. At present, Panama is a discreet producer of coffee globally due to it is a very small country. However, a mix of favorable environmental conditions (altitude, precipitation, temperature, relative humidity, inter alia) makes the country ideal for the production of high-quality coffee (Cherigo et al., 2012; 2015). The main coffee production area is located in the province of Chiriqui. In this region coffee production is an activity of high economic value. Therefore, the harvest efficiency of this crop is crucial for farmers. Unfortunately, every year coffee production is widely affected by several factors including pests, weeds and climate change, among others.

\begin{abstract}
Worldwide, coffee is one of the most highly consumed and produced crops. Coffee production is a significant activity in the Panamanian economy, mainly in rural communities and among indigenous groups in the Chiriquí province highlands. Weeds growing alongside coffee plants can provoke considerable economic losses for producers by interfering with the growth, development and yield of coffee crop in cultivated areas. Designing an effective program to control weeds depends on identifying the different species found in the coffee plantations. The objective of this study was to assess the biological diversity and negative allelopathic potential of weeds in a coffee field to generate enough information that would better allow farmers to control them. As a result, we identified forty-two different species of weeds in all sampling transects within the study area. Emilia sonchifolia and Impatiens walleriana were the most abundant. In respect to phytotoxic activity, Emilia sonchifolia and He capitata showed the highest activity against the seed germination of dicotyledonous species Amaranthus hypochondriacus, exhibiting $\mathrm{IC}_{50}$ values of 160 and $178 \mu \mathrm{g} \mathrm{mL} \mathrm{L}^{-1}$, respectively. Finally, we proceeded to evaluate the organic extracts of two coffee weeds in a panel of bioassays to demonstrate to the farmers that weeds may also have useful applications for human health. pyramidatum displayed inhibition of the $\alpha-$ alucosidase enzyme. These rults allow us to propose a rational and systematic management of coffee weeds.
\end{abstract}

Keywords: Weeds, Coffee, Allelopathic Activity, Control, Abundance
Weeds provoke considerable economic losses for coffee producers by interfering with the growth and development of the coffee plants in the fields. Weeds directly reduce coffee crop yields by competing for nutrients, sunlight, water and negative allelopathic effects. Also, they can contain pests and pathogens which can also decrease the yield and quality of coffee (De Graaff, 1986; Radosevich et al., 1997; Cherigo et al., 2012).

Weed competition against coffee plants could be minimized by reducing or eliminating weeds in the cultivated fields. Most traditional farmers in the Chiriqui Province still work under the paradigm that weeds are plants that only cause damage to crops, so they often eliminate them completely. However, recent studies have shown that different weed species have 
potentially useful applications for humans. For example, some weeds can protect against other weeds when they are used as cover crops (Altieri, 1995). In addition, there are other weeds with ethno medical uses (Pani et al., 2002; Panda et al., 2014). Therefore, it is important to generate information that would allow farmers to distinguish between potentially useful weeds from those having only negative effects for their rational or systematic elimination (Altieri, 1995; Cherigo et al., 2012). The identification of the allelopathic properties of weeds, especially those related to the inhibition of plant germination and growth (phytotoxins), is of significant relevance because some phytotoxins could be efficient substitutes for highly toxic synthetic herbicides as those currently used in developing countries (Bhadoria, 2011).

We suggest that one of the first steps towards more sustainable crop production is to gain complete knowledge of the plant species diversity within the defined growing area. Therefore, studies to generate information about biological diversity and dynamics of weeds within crop fields are necessary for their rational and systematic control. Examples of this can be seen in studies performed in countries like Brazil (Dedeca, 1959; Ronchi and Silva, 2006), Cuba (Caro et al., 1990), Honduras (Ordoñez et al., 2000), Kenya (Anonymous, 1992), Mexico (Braver, 1957), Nicaragua (Aguilar et al., 2003) and Papua Nueva Guinea (Byrne, 1984), where farmers have developed effective weed control programs from the identification of the different species found in their coffee plantations. These studies highlight that appropriate methods for handling coffee weeds should be based on a better understanding of the biology and population dynamics of different species.

Our research group proceeded to record the different types of weeds that grow in the coffee plantations in Santa Clara (Chiriquí). We also evaluated the phytotoxic potential of the weeds using a standardized method for determining if the identified weeds provoke adverse effects by chemical competition (production of phytotoxic compounds) or/and by competing for nutrients, light and available water in soil (Cherigo et al., 2012 ; 2015). Finally, we proceeded to evaluate the organic extracts from Blechum pyramidatum and Borreria verticillata in a panel of bioassays to demonstrate to farmers that weeds may also have useful applications to human health. All the information generated will serve to propose to local farmers changing their conventional strategies of elimination of weeds by others approaches systematic and rationally designed.

\section{Materials and Methods}

\section{Study area and Sampling}

An important part of our study was to select a representative area that possesses the prevailing growing conditions (altitude, soil, temperature, among others) of the Panamanian coffee fields. We located an appropriate region for this study in the town of Santa Clara. Once we got the required permits (from the owner and the Panamanian government), we proceeded with our study.

The study field was divided into 12 altitudinal floors; each altitudinal section was established by an increase of 50 meters' elevation above sea level (m.a.s.l.). The first altitudinal floor was located at 950 meters and the last at 1500 meters. To facilitate the recording of weeds, we subdivided each altitudinal floor in 3 transects of equal length and every two meters all existing varieties of weeds were collected and recorded. Also, the geographic location information of each collected weed was recorded using Global Positioning System (GPS) coordinates (Table 1).

According to standard protocols, we considered "abundant" all weeds that were found at least twice in two of three installed transects per floor. All obtained data is in Table 2, abundant weeds were marked with "X," and weeds found only once or absent were marked with "0". The number of altitudinal floors marked with "X" was divided by the total number of transects (12) to generate the weed Abundance Index (AI), which reflects the percentage of weed appearance. To illustrate the diversity of weeds that occurred for each altitudinal floor, we proceeded to generate the Altitudinal Floor Index (AFI), which was calculated by taking the number of species found at each altitudinal floor and dividing it by the total number of species found in the 12 floors (Table 2).

\section{Taxonomic Identification}

The identification of weeds was pursued using taxonomic keys from the Flora of Panama and the Mesoamerican Flora. Weed identification was also confirmed employing herbarium specimens from the University of Panama. Finally, nomenclature of all collected species was verified using the TROPICOS database from Missouri Botanical Garden and Vascular Plants of Panama catalog.

\section{Plant Material for Phytotoxic Assays}

Approximately 100 grams of the fresh aerial parts of each of the different coffee weeds species were randomly collected in the sampling area. Samples were stored in black bags and immediately transported to the laboratory. The samples were rinsed with water and placed to dry at room temperature. Once dried, the samples were pulverized and stored for further processing.

\section{Organic Extracts Preparation}

To obtain a maximum amount of organic constituents the extract from each plant was prepared five times by a maceration process using a mixture of ethyl acetatemethanol (1:1). The mixture of solvents was concentrated using a rotary evaporator (Laborota 4010, Germany) until a semisolid paste of crude extract was obtained. This extract was stored at $-4^{\circ} \mathrm{C}$ until further use (Cherigo et al., 2012; 2015). 
Table 1. Species of weeds collected and identified within the studied growing area

\begin{tabular}{|c|c|c|}
\hline Code & Specie & Coordinates \\
\hline LIJ 873 & Acalypha villosa & $\mathrm{N} 8^{\circ} 51^{\prime} 24^{\prime \prime} \mathrm{W} 81^{\circ} 47^{\prime} 52^{\prime \prime}$ \\
\hline LIJ 856 & Achyranthes aspera & $\mathrm{N} 8^{\circ} 50^{\prime} 45^{\prime}, \mathrm{W} 81^{\circ} 46^{\prime} 42^{\prime}$, \\
\hline LIJ 1012 & Asclepias curassavica & $\mathrm{N} 8^{\circ} 50^{\prime} 45^{\prime}$ ' W $81^{\circ} 46^{\prime} 52^{\prime}$, \\
\hline LIJ 1023 & Baccharis trinervis & $\mathrm{N} 8^{\circ} 50^{\prime} 45^{\prime}, \mathrm{W} 81^{\circ} 46^{\prime} 52^{\prime \prime}$ \\
\hline LIJ 883 & Bidens pilosa & $\mathrm{N} 8^{\circ} 50^{\prime} 05^{\prime}, \mathrm{W} 81^{\circ} 46^{\prime} 52^{\prime}$, \\
\hline LIJ 887 & Blechum pyramidatum & N $8^{\circ} 50^{\prime} 05^{\prime}, \mathrm{W} 81^{\circ} 46^{\prime} 52^{\prime}$, \\
\hline LIJ 874 & Borreria verticillata & $\mathrm{N} 8^{\circ} 51^{\prime} 13^{\prime \prime}$ W $81^{\circ} 46^{\prime} 55^{\prime}$, \\
\hline LIJ 1009 & Browallia americana & $\mathrm{N} 8^{\circ} 51^{\prime} 13^{\prime \prime} \mathrm{W} 81^{\circ} 34^{\prime} 76^{\prime \prime}$ \\
\hline LIJ 1007 & Centradenia inaequilateralis & $\mathrm{N} 8^{\circ} 51^{\prime} 13^{\prime \prime}, \mathrm{W} 81^{\circ} 46^{\prime} 55^{\prime}$, \\
\hline LJJ 892 & Commelina diffusa & $\mathrm{N} 8^{\circ} 50^{\prime} 05^{\prime}, \mathrm{W} 81^{\circ} 46^{\prime} 62^{\prime \prime}$ \\
\hline LIJ 1020 & Cuphea sp. & $\mathrm{N} 8^{\circ} 50^{\prime} 05^{\prime}, \mathrm{W} 81^{\circ} 46^{\prime} 62^{\prime}$, \\
\hline LIJ 871 & Cuphea calophylla & $\mathrm{N} 8^{\circ} 50^{\prime} 15^{\prime}, \mathrm{W} 81^{\circ} 46^{\prime} 52^{\prime \prime}$ \\
\hline LIJ 1015 & Elephantopus mollis & $\mathrm{N} 8^{\circ} 50^{\prime} 16^{\prime}$ 'W $81^{\circ} 46^{\prime} 41^{\prime}$, \\
\hline LIJ 885 & Elephantopus mollis & $\mathrm{N} 8^{\circ} 50^{\prime} 05^{\prime}, \mathrm{W} 81^{\circ} 46^{\prime} 51^{\prime}$, \\
\hline LIJ 869 & Emilia sonchifolia & $\mathrm{N} 8^{\circ} 50^{\prime} 25^{\prime}$ 'W $81^{\circ} 46^{\prime} 52^{\prime}$, \\
\hline LIJ 1005 & Erechtites hieracifolia & 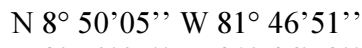 \\
\hline LIJ 862 & Hyptis brachiata & $\mathrm{N} 8^{\circ} 50^{\prime} 05^{\prime}, \mathrm{W} 81^{\circ} 46^{\prime} 52^{\prime}$, \\
\hline LIJ 852 & Hyptis capitata & $\mathrm{N} 8^{\circ} 50^{\prime} 05^{\prime}, \mathrm{W} 81^{\circ} 46^{\prime} 52^{\prime}$, \\
\hline LIJ 1030 & Hyptis conferta & $\mathrm{N} 8^{\circ} 50^{\prime} 13^{\prime \prime} \mathrm{W} 81^{\circ} 46^{\prime} 54^{\prime \prime}$ \\
\hline LIJ 1029 & Hyptis pectinata & $\mathrm{N} 8^{\circ} 50^{\prime} 08^{\prime}, \mathrm{W} 81^{\circ} 47^{\prime} 01^{\prime}$, \\
\hline LIJ 889 & Impatiens walleriana & $\mathrm{N} 8^{\circ} 50^{\prime} 05^{\prime}, \mathrm{W} 81^{\circ} 46^{\prime} 52^{\prime}$, \\
\hline LIJ 1018 & Iresine diffusa & $\mathrm{N} 8^{\circ} 50^{\prime} 15^{\prime}, \mathrm{W} 81^{\circ} 46^{\prime} 12^{\prime}$, \\
\hline LIJ 853 & Lactuca graminifolia & $\mathrm{N} 8^{\circ} 50^{\prime} 52^{\prime}, \mathrm{W} 81^{\circ} 46^{\prime} 52^{\prime \prime}$ \\
\hline LIJ 896 & Ludwigia erecta & $\mathrm{N} 8^{\circ} 50^{\prime} 05^{\prime}, \mathrm{W} 81^{\circ} 46^{\prime} 23^{\prime \prime}$ \\
\hline LIJ 893 & Mimosa pudica & $\mathrm{N} 8^{\circ} 50^{\prime} 04^{\prime \prime} \mathrm{W} 81^{\circ} 46^{\prime} 56^{\prime}$, \\
\hline LIJ 1033 & Monnina sylvatica & $\mathrm{N} 8^{\circ} 51^{\prime} 34^{\prime \prime}$ W $81^{\circ} 45^{\prime} 12^{\prime}$, \\
\hline LIJ 857 & Oxalis sp. & $\mathrm{N} 8^{\circ} 51^{\prime} 15^{\prime}, \mathrm{W} 81^{\circ} 44^{\prime} 59^{\prime}$, \\
\hline LIJ 888 & Phyllanthus sp. & $\mathrm{N} 8^{\circ} 50^{\prime} 05^{\prime}, \mathrm{W} 81^{\circ} 46^{\prime} 52^{\prime \prime}$ \\
\hline LIJ 1032 & Piriqueta sp. & $\mathrm{N} 8^{\circ} 50^{\prime} 05^{\prime}, \mathrm{W} 81^{\circ} 46^{\prime} 52^{\prime \prime}$ \\
\hline LIJ 1016 & Polygala longicaulis & $\mathrm{N} 8^{\circ} 51^{\prime} 95^{\prime}, \mathrm{W} 81^{\circ} 43^{\prime} 32^{\prime \prime}$ \\
\hline LIJ 877 & Polygala panamensis & $\mathrm{N} 8^{\circ} 50^{\prime} 05^{\prime}, \mathrm{W} 81^{\circ} 47^{\prime} 52^{\prime}$, \\
\hline LIJ 880 & Pseudelephantopus spicatus & $\mathrm{N} 8^{\circ} 50^{\prime} 05^{\prime}, \mathrm{W} 81^{\circ} 46^{\prime} 52^{\prime}$, \\
\hline LIJ 891 & Rumex crispus & $\mathrm{N} 8^{\circ} 50^{\prime} 06^{\prime}, \mathrm{W} 81^{\circ} 46^{\prime} 2^{\prime \prime}$ \\
\hline LIJ 1039 & Salvia occidentalis & $\mathrm{N} 8^{\circ} 50^{\prime} 76^{\prime}, \mathrm{W} 81^{\circ} 46^{\prime} 82^{\prime}$, \\
\hline LIJ 878 & Scrophulariaceae & $\mathrm{N} 8^{\circ} 50^{\prime} 05^{\prime}, \mathrm{W} 81^{\circ} 46^{\prime} 52^{\prime}$, \\
\hline LIJ 1042 & Sida acuta & $\mathrm{N} 8^{\circ} 50^{\prime} 05^{\prime}, \mathrm{W} 81^{\circ} 46^{\prime} 52^{\prime}$, \\
\hline LIJ 881 & Sida rhombifolia & $\mathrm{N} 8^{\circ} 50^{\prime} 32^{\prime \prime} \mathrm{W} 81^{\circ} 46^{\prime} 22^{\prime \prime}$ \\
\hline LIJ 884 & Solanum nigrescens & N $8^{\circ} 50^{\prime} 06^{\prime}, \mathrm{W} 81^{\circ} 46^{\prime} 87^{\prime}$, \\
\hline LIJ 882 & Sonchus asper & $\mathrm{N} 8^{\circ} 50^{\prime} 05^{\prime}, \mathrm{W} 81^{\circ} 46^{\prime} 23^{\prime}$, \\
\hline LIJ 865 & Spermacoce sp. & $\mathrm{N} 8^{\circ} 50^{\prime} 89^{\prime}, \mathrm{W} 81^{\circ} 46^{\prime} 43^{\prime}$, \\
\hline LIJ 1002 & Spigelia humboldtiana & $\mathrm{N} 8^{\circ} 50^{\prime} 90^{\prime \prime} \mathrm{W} 81^{\circ} 47^{\prime} 52^{\prime \prime}$ \\
\hline LIJ 1001 & Tradescantia commelinoides & $\mathrm{N} 8^{\circ} 50^{\prime} 34^{\prime \prime} \mathrm{W} 81^{\circ} 47^{\prime} 32^{\prime \prime}$ \\
\hline LIJ 875 & Youngia japonica & 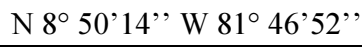 \\
\hline
\end{tabular}

\section{Inhibition of Radical Elongation of Amaranthus Hypochondriacus}

The growth inhibitory activity of the extracts on seedlings of $A$. hypochondriacus was evaluated using the Petri dish radicle elongation and germination bioassay (Mata et al., 1998; Cherigo et al., 2012).

Briefly, organic extracts were dissolved in ethyl acetate and we prepared dilutions with final concentrations of 10,100 and $1000 \mu \mathrm{g} \mathrm{m}^{-1}$. One $\mathrm{mL}$ of the test solution was added to a Petri dish containing a filter paper disc; then the solvent was evaporated in an extraction hood. After complete evaporation of the solvent, $3 \mathrm{~mL}^{-1}$ of distilled water and ten Amaranthus hypochondriacus seeds were added to the filter paper. This procedure was performed in triplicate (30 seeds for each evaluated extract). Petri dishes were incubated in the dark at $28^{\circ} \mathrm{C}$ for $24 \mathrm{~h}$. After this time, the radicular growth of each seed was measured. Petri Dishes with 2,4-D were used as a positive control. As a negative control, we used Petri dishes with treatment but without extract and also Petri dishes with seeds without treatment.

The results were analyzed by ANOVA $(\mathrm{p}<0.05)$ and $\mathrm{IC}_{50}$ values were calculated by Probit analysis based on the percent of radicular growth or germination inhibition. 
Table 2. Abundance of weeds through altitudinal floors

\begin{tabular}{|c|c|c|c|c|c|c|c|c|}
\hline Code & $1-2$ & $3-4$ & $5-6$ & $7-8$ & $9-10$ & $11-12$ & $\mathrm{~A}$ & $\mathrm{AI}$ \\
\hline $\begin{array}{l}\text { LIJ } 873 \\
\end{array}$ & X-X & X-X & X-X & X-0 & $0-\mathrm{X}$ & $0-0$ & 8 & 0.66 \\
\hline LIJ 856 & $X-0$ & $X-X$ & $0-0$ & $0-0$ & $0-0$ & $0-0$ & 3 & 0.25 \\
\hline LIJ 1012 & $0-X$ & $X-X$ & $X-0$ & $X-X$ & $0-0$ & $0-0$ & 6 & 0.50 \\
\hline LIJ 1023 & $0-0$ & $0-\mathrm{X}$ & $\mathrm{X}-0$ & $X-0$ & $X-0$ & $\mathrm{X}-0$ & 5 & 0.41 \\
\hline LIJ 883 & $X-X$ & $\mathrm{X}-0$ & $\mathrm{X}-0$ & $0-0$ & $0-0$ & $0-0$ & 4 & 0.30 \\
\hline LIJ 874 & $X-X$ & $0-0$ & $0-\mathrm{X}$ & $0-0$ & $0-0$ & $0-0$ & 3 & 0.25 \\
\hline LIJ 1009 & $X-X$ & $0-\mathrm{X}$ & $0-\mathrm{X}$ & $X-X$ & $\mathrm{X}-0$ & $0-0$ & 7 & 0.58 \\
\hline LIJ 1007 & $0-0$ & $0-0$ & $0-0$ & $X-0$ & $\mathrm{X}-0$ & $X-X$ & 4 & 0.33 \\
\hline LJJ 892 & $0-0$ & $0-0$ & $0-0$ & $X-X$ & $X-0$ & $0-\mathrm{X}$ & 3 & 0.25 \\
\hline LIJ 1020 & $0-\mathrm{X}$ & $X-X$ & $X-X$ & $0-0$ & $0-0$ & $0-0$ & 5 & 0.41 \\
\hline LIJ 871 & $0-0$ & $0-0$ & $0-0$ & $X-0$ & $0-0$ & $0-0$ & 1 & 0.08 \\
\hline LIJ 1015 & $0-0$ & $0-\mathrm{X}$ & $X-X$ & $0-0$ & $0-0$ & $0-0$ & 3 & 0.25 \\
\hline LIJ 885 & $X-X$ & $0-\mathrm{X}$ & $X-X$ & $0-0$ & $X-X$ & $\mathrm{X}-0$ & 8 & 0.66 \\
\hline LIJ 869 & $X-X$ & $X-X$ & $X-X$ & $X-X$ & $X-0$ & $0-\mathrm{X}$ & 10 & 0.83 \\
\hline LIJ 1005 & $0-0$ & $0-X$ & $X-0$ & $X-X$ & $X-0$ & $0-0$ & 5 & 0.41 \\
\hline LIJ 862 & $0-X$ & $X-X$ & $X-X$ & $X-0$ & $0-0$ & $0-0$ & 6 & 0.50 \\
\hline LIJ 852 & $X-0$ & $X-X$ & $X-X$ & $X-X$ & $X-0$ & $0-0$ & 8 & 0.66 \\
\hline LIJ 1030 & $0-0$ & $0-0$ & $X-X$ & $X-X$ & $0-0$ & $0-0$ & 4 & 0.33 \\
\hline LIJ 1029 & $X-X$ & $X-X$ & $0-0$ & $X-0$ & $0-0$ & $\mathrm{X}-0$ & 6 & 0.50 \\
\hline LIJ 889 & $X-X$ & $X-X$ & $X-X$ & $X-X$ & $X-X$ & $0-0$ & 10 & 0.83 \\
\hline LIJ 1018 & $X-0$ & $0-0$ & $0-0$ & $X-0$ & $0-0$ & $\mathrm{X}-0$ & 3 & 0.25 \\
\hline LIJ 853 & $0-X$ & $X-X$ & $X-X$ & $X-X$ & $X-0$ & $X-X$ & 8 & 0.66 \\
\hline LIJ 896 & $0-X$ & $0-\mathrm{X}$ & $X-X$ & $X-0$ & $0-0$ & $0-0$ & 5 & 0.41 \\
\hline LIJ 893 & $X-X$ & $X-X$ & $X-X$ & $0-0$ & $0-0$ & $0-0$ & 6 & 0.50 \\
\hline LIJ 1033 & $0-0$ & $0-\mathrm{X}$ & $X-X$ & X-0 & $0-0$ & $0-0$ & 3 & 0.25 \\
\hline LIJ 857 & $0-X$ & $X-X$ & $X-X$ & $0-0$ & $0-0$ & $0-0$ & 5 & 0.41 \\
\hline LIJ 888 & $0-\mathrm{X}$ & $X-X$ & $X-0$ & $\mathrm{X}-0$ & $X-0$ & $0-0$ & 6 & 0.50 \\
\hline LIJ 1032 & $0-\mathrm{X}$ & $X-X$ & $0-0$ & $0-X$ & $X-0$ & $0-0$ & 5 & 0.41 \\
\hline LIJ 1016 & $0-X$ & $X-X$ & $\mathrm{X}-0$ & $X-X$ & $0-\mathrm{X}$ & $0-0$ & 7 & 0.58 \\
\hline LIJ 877 & $0-X$ & $0-\mathrm{X}$ & $0-0$ & $\mathrm{X}-0$ & $0-0$ & $0-0$ & 3 & 0.25 \\
\hline LIJ 880 & $X-X$ & $X-X$ & $0-\mathrm{X}$ & $X-0$ & $\mathrm{X}-0$ & $0-0$ & 7 & 0.58 \\
\hline LIJ 891 & $0-X$ & $X-X$ & $X-X$ & $X-X$ & $X-0$ & $0-0$ & 8 & 0.66 \\
\hline LIJ 1039 & $0-0$ & $0-0$ & $0-\mathrm{X}$ & $0-X$ & $X-X$ & $\mathrm{X}-0$ & 5 & 0.41 \\
\hline LIJ 878 & $0-0$ & $0-\mathrm{X}$ & $0-0$ & $0-0$ & $0-0$ & $0-0$ & 1 & 0.11 \\
\hline LIJ 1042 & $0-0$ & $0-0$ & $0-0$ & $0-0$ & $\mathrm{X}-0$ & $X-X$ & 3 & 0.25 \\
\hline LIJ 881 & $0-X$ & $X-X$ & $0-\mathrm{X}$ & $X-0$ & $0-0$ & $0-0$ & 5 & 0.41 \\
\hline LIJ 884 & $0-X$ & $X-X$ & $X-X$ & $0-0$ & $0-0$ & $0-0$ & 5 & 0.41 \\
\hline LIJ 882 & $0-X$ & $X-X$ & $X-X$ & $X-0$ & $X-0$ & $0-0$ & 7 & 0.58 \\
\hline LIJ 865 & $0-X$ & $X-X$ & $X-X$ & $0-0$ & $0-X$ & $X-X$ & 8 & 0.66 \\
\hline LIJ 1002 & $0-0$ & $0-X$ & $X-X$ & $X-0$ & $0-0$ & $0-0$ & 4 & 0.33 \\
\hline LIJ 1001 & $0-X$ & $0-\mathrm{X}$ & $X-X$ & $0-0$ & $0-0$ & $0-0$ & 4 & 0.66 \\
\hline LIJ 875 & $0-0$ & $X-X$ & $X-X$ & $\mathrm{X}-0$ & $0-0$ & $0-0$ & 5 & 0.41 \\
\hline AFI & $0.30-0.61$ & $0.57-0.78$ & $0.64-0.52$ & $0.64-0.30$ & $0.40-0.14$ & $0.21-0.14$ & & \\
\hline
\end{tabular}

*A: Total Abundance, AI: Abundance Index, AFI: Abundance Floor Index

\section{Malaria and $\alpha$-Glucosidase Bioassays}

Malaria bioassays were performed as previously reported by us using chloroquine as a positive control (Cherigo et al. 2012). $\alpha$-glucosidase enzyme bioassays were conducted following the protocol of Lopez et al. (2015) using acarbose as a positive control.

\section{Results}

\section{Sampling and Identification of Weeds}

A total of 42 different species of weeds were collected and identified among the evaluated crops. Table 1 lists all species together with their location or GPS coordinates.

The abundance of identified species along marked altitudinal levels is detailed in Table 2. The most abundant species -Emilia sonchifolia, Impatiens walleriana, Elephantopus mollis, Hyptis capitata, Lactuca graminifolia, Rumex crispus and Spermacocce sp.- were found in more than 7 altitudinal floors ( $\geq 66 \%$ presence). Among these, the first two species are the most abundant weeds with an abundance index of 0.83. Four species -Borreria verticillata, Polygala longicaulis, Pseudelephantopus spicatus and Sonchus asper- were present in $58 \%$ of the sampled floors. 


\section{Allelopathic Potential}

$\mathrm{IC}_{50}$ values for the 42 evaluated extracts are shown in Table 3. The degree of extract phytotoxicity was determined based on the range in which the $\mathrm{IC}_{50}$ values were encountered using the following categories: very phytotoxic $\left(\mathrm{IC}_{50}<100 \mu \mathrm{g} \mathrm{mL} \mathrm{L}^{-1}\right)$, phytotoxic $\left(100<\mathrm{IC}_{50}<500\right.$ $\left.\mu \mathrm{g} \mathrm{mL} \mathrm{m}^{-1}\right)$, moderately phytotoxic $\left(500<\mathrm{IC}_{50}<1000\right)$ and non-phytotoxic $\left(\mathrm{IC}_{50}>1000 \mu \mathrm{g} \mathrm{mL} \mathrm{m}^{-1}\right)$. In practical terms, $\mathrm{IC}_{50}$ values within the very phytotoxic category or very close to this range are those that could cause a markedly adverse effect on coffee plants.

The extracts that showed the highest phytotoxic activity were from Emilia sonchifolia and Hyptis capitata exhibiting $\mathrm{IC}_{50}$ values of 160 and $178 \mu \mathrm{g}$ $\mathrm{mL}^{-1}$, respectively. Extracts from Oxalis sp., Commelina diffusa, Scrophullariaceae sp., Salvia occidentalis, Bidens pilosa and Hyptis pectinate also displayed activity into the phytotoxic range. On the other hand, Baccharis trinervis, Hyptis conferta, Rumex crispus, Hyptis brachiate, Asclepias curassavica, Phyllantus sp., Cuphea sp., Youngia japonica, Blechum pyramidatum, Sonchus asper, Cuphea calophylla, Achyranthes aspera, Lactuca gramifolia, Sida acuta, Ludwigia erecta, Iresine diffusa, Spermacocce sp. and Sida rhombifolia showed activity into de moderate phytotoxicity range. Finally, Acalypha villosa, Borreria verticillata, Browallia americana, Centradenia inaequilateralis, Elephantopus mollis, Erechtites hieracifolia, Impatiens walleriana, Mimosa pudia, Monnina sylvatica, Piriqueta sp., Polygala longicaulis, Polygola panamensis, Pseudelephantopus spicatus, Solanum nigrescens, Spigelia humboldtiana and Tradescantia commelinoides presented a phytotoxicity above $1000 \mu \mathrm{g} \mathrm{mL}^{-1}$ so that for practical purposes are considered inactive.

Table 3. Phytotoxic activity of coffee weeds

\begin{tabular}{|c|c|c|}
\hline Specie & $\mathrm{IC}_{50}(\mu \mathrm{g} / \mathrm{mL})$ & Category \\
\hline Acalypha villosa & $>1000$ & Non-phytotoxic \\
\hline Achyranthes aspera & 843 & Moderately phytotoxic \\
\hline Asclepias curassavica & 670 & Moderately phytotoxic \\
\hline Baccharis trinervis & 559 & Moderately phytotoxic \\
\hline Bidens pilosa & 470 & Phytotoxic \\
\hline Blechum pyramidatum & 794 & Moderately phytotoxic \\
\hline Borreria verticillata & $>1000$ & Non-phytotoxic \\
\hline Browallia americana & $>1000$ & Non-phytotoxic \\
\hline Centradenia inaequilateralis & $>1000$ & Non-phytotoxic \\
\hline Commelina diffusa & 395 & Phytotoxic \\
\hline Cuphea sp. & 763 & Moderately phytotoxic \\
\hline Cuphea calophylla & 821 & Moderately phytotoxic \\
\hline Elephantopus mollis & $>1000$ & Non-phytotoxic \\
\hline Elephantopus mollis & $>1000$ & Non-phytotoxic \\
\hline Emilia sonchifolia & 160 & Phytotoxic \\
\hline Erechtites hieracifolia & $>1000$ & Non-phytotoxic \\
\hline Hyptis brachiata & 634 & Moderately phytotoxic \\
\hline Hyptis capitata & 178 & Phytotoxic \\
\hline Hyptis conferta & 564 & Moderately phytotoxic \\
\hline Hyptis pectinata & 497 & Phytotoxic \\
\hline Impatiens walleriana & $>1000$ & Non-phytotoxic \\
\hline Iresine diffusa & 938 & Moderately phytotoxic \\
\hline Lactuca graminifolia & 872 & Moderately phytotoxic \\
\hline Ludwigia erecta & 912 & Moderately phytotoxic \\
\hline Mimosa pudica & $>1000$ & Non-phytotoxic \\
\hline Monnina sylvatica & $>1000$ & Non-phytotoxic \\
\hline Oxalis sp. & 387 & Phytotoxic \\
\hline Phyllanthus sp. & 675 & Moderately phytotoxic \\
\hline Piriqueta sp. & $>1000$ & Non-phytotoxic \\
\hline Polygala longicaulis & $>1000$ & Non-phytotoxic \\
\hline Polygala panamensis & $>1000$ & Non-phytotoxic \\
\hline Pseudelephantopus spicatus & $>1000$ & Non-phytotoxic \\
\hline Rumex crispus & 619 & Moderately phytotoxic \\
\hline Salvia occidentalis & 432 & Phytotoxic \\
\hline Scrophulariaceae & 398 & Phytotoxic \\
\hline Sida acuta & 884 & Moderately phytotoxic \\
\hline Sida rhombifolia & 986 & Moderately phytotoxic \\
\hline Solanum nigrescens & $>1000$ & Non-phytotoxic \\
\hline Sonchus asper & 803 & Moderately phytotoxic \\
\hline Spermacoce sp. & 958 & Moderately phytotoxic \\
\hline Spigelia humboldtiana & $>1000$ & Non-phytotoxic \\
\hline Tradescantia commelinoides & $>1000$ & Non-phytotoxic \\
\hline Youngia japonica & 774 & Moderately phytotoxic \\
\hline
\end{tabular}




\section{Identification of Alternative Biological Properties in Coffee Weeds}

To achieve this objective, we selected the two weeds which had generated the largest quantities of organic extracts, B. verticillata and B. pyramidatum. In order to get evidence of useful applications of both weeds, we evaluated their extracts utilizing a panel of bioassays that included antiparasitic (Leishmania donovani, Plasmodium falciparum and Tripanosoma cruzi), anticancer (MCF-7 cell) and hypoglycemic $(\alpha-$ glucosidase inhibition) activities. As a result, $B$. verticillata sample showed antiparasitic activity $(65 \%$ growth inhibition at $10 \mu \mathrm{g} \mathrm{mL}^{-1}$ ) against Plasmodium falciparum. On the other hand, the organic extract from B. pyramidatum showed moderate inhibition $(63 \%$ of inhibition) of the $\alpha$-glucosidase enzyme. Both samples were totally inactive in all the other bioassays.

\section{Discussion}

This study is the first to identify weeds that grow in some of Panama's fields of coffee crop production. It is important to point out that the total number of different species detected was lower than that reported for other countries, although the type of weeds is similar. The number of weeds per altitudinal floor declined strongly with increasing altitude. At floors $\leq$ 1200 m.a.s.l. (six lower floors) there were 147 records from weeds marked as abundant in this study while there were only 79 records at sites $<1200$ m.a.s.l. (six upper floors). These findings were corroborated by the analysis of AFI index, where it was observed that the greatest AFIs were produced in the altitudinal floors between 1050 and 1100 meters' elevation (AFI values of 0.57 and 0.78 , respectively). At the highest floors, the AFI index value tended to decrease finishing with a value of 0.14 in the last altitudinal floor. Among the less abundant species, Browallia americana, Centradenia inaequilateralis, Cuphea sp. and Sida acuta were recorded only in the six upper floors while Solanum nigrescens, Tradescantia commelinoides, Scrophullariaceae sp., Oxalis sp., Mimosa pudica, Cuphea calophylla, Commelina diffusa, Blechum pyramidatum, Bidens pilosa and Achyranthes aspera were recorded only in the six lower floors.

The phytotoxic activity of organic extracts from weeds was evaluated against the seed germination and initial radical elongation of dicotyledonous species Amaranthus hypochondriacus (Amaranthaceae). Several species of the genus Amaranthus are broadleaved weeds that are commonly associated with coffee plantations (Njoroge, 1994). On the other hand, Amaranthus is one of the model genus widely standardized for phytotoxicity studies of plant extracts
(Anaya et al., 1990; Mata et al., 1998; Cherigo et al., 2012). Therefore, this species was considered adequate as target weed for our allelopathic evaluation. In addition, the seeds of this weed present a fast, uniform and consistent germination, which facilitates the implementation of this bioassay. The crude ethyl acetate-MeOH (1:1) extracts from all coffee weeds were prepared. This solvent mixture was used to obtain the greater amount of both hydrophilic and lipophilic plant components. The phytotoxic activity identification of weeds extracts could be beneficial in two different ways: (1) in the identification of the weeds that can cause more chemical damage to commercial plants and (2) in the determination for phytotoxins with potential use as selective bioherbicide mainly against certain specific types of weeds. According to $\mathrm{IC}_{50}$ values, Emilia sonchifolia and Hyptis capitata are the weeds that could cause damage to coffee plants in the plantations due to their allelopathic potential. It is highly likely that the other 40 species only can interfere with coffee trees by competition for nutrients and water present in the soil.

In traditional agricultural practices, weeds are seen as entirely undesirable agents and farmers want to eliminate them at any cost, but farmers do not know that even though weeds can cause significant economic losses in their crops, they can also have beneficial applications for humans. We just have to remember that plants have also been prolific producers of pharmacological and agrochemical important metabolites (Khalid et al., 2002; Cragg and Newman, 2013; Pino et al., 2013; Atanasov et al., 2015). For this reason, searching for biological properties in weeds can also allow us to detect potential beneficial applications for humans. So, we proceeded to evaluate the organic extracts from $B$. verticillata and $B$. pyramidatum in a panel of bioassays that included antiparasitic (Leishmania donovani, Plasmodium falciparum and Tripanosoma cruzi), anticancer (MCF7 cell) and hypoglycemic ( $\alpha$-glucosidase inhibition) activities, to get evidence of useful applications of this weed. $B$. verticillata sample showed selective activity (65\% growth inhibition at $10 \mu \mathrm{g} \mathrm{mL} \mathrm{mL}^{-1}$ ) against Plasmodium falciparum. Before this study, the antimalarial activity of this plant has never been reported and no antiprotozoal metabolites from $B$. verticillata have been published. On the other hand, the organic extract from B. pyramidatum showed $63 \%$ of inhibition of the $\alpha$-glucosidase enzyme. $\alpha$ glucosidase hydrolyzes starch and disaccharides to release glucose and high levels of sugar are related to diabetes mellitus. Inhibition of this enzyme is one of the key mechanisms to regulate blood sugar levels, so this plant could be an attractive hypoglycemic agent. 


\section{Conclusion}

Weed diversity found in Panamanian coffee crops is less than that reported in coffee production in other countries. Forty-two different species were found, most of which have been previously reported as common weeds in coffee plantations. Most collected weeds had a relatively high abundance and their IA was above 0.33 , although there were also a few weed species that had an IA equal to or below 0.33 (with very low abundance). The most abundant weeds belong to Asteraceae family, particularly Emilia sonchifolia and Impatiens walleriana. In the allelopathic bioassays, only eight of the forty-two species showed marked phytotoxicity. In fact, only Emilia sonchifolia and Hyptis capitata showed values of $\mathrm{IC}_{50} \mathrm{~S}$ that could cause a marked in vivo negative allelopathic effect against coffee plants. This suggests that the weeds identified in this study are more likely to cause more damage by their physical competition than by their negative allelopathic effects. Biological evaluations of $B$. pyramidatum and $B$. verticillata extracts showed that these weeds possess hypoglycemic and antimalaric activities. The information generated in this study offers valuable tools that will help us to propose to the farmers a rational, systematic and selective elimination of weeds because, as we have shown, weeds are not completely harmful to coffee plants and they can have useful applications for the human health.

\section{Acknowledgement}

We gratefully acknowledge the Government of Panama (ANAM) for granting permission to make the weed collections. To Alberto E. Morales (Department of Anthropology at the University of California, Irvine) for the spelling and grammar revision.

\section{Funding Information}

This work was supported by the National Secretariat for Science and Technology of Panama (SENACYT, grant FID 10-074). LC and SM-L were supported by funds from the National Research System (SNI, SENACYT) [SNI-1472016 and SNI-145-2016, respectively].

\section{Author's Contributions}

Lilia Cherigo: Conducted research (preparation of extracts and allelopathic evaluations), analysis of results, manuscript revision.

Jorge Lezcano: Conducted research (Made Collection and identification of weed species), analysis of results, manuscript revision.

Sergio Martinez-Luis: Provided leadership and coordinated the implementation of research work, conducted research (preparation of extracts, allelopathic evaluations, bioassays) analyzed and interpreted the study findings.

\section{Ethics}

This article is original and contains unpublished material. The corresponding author confirms that all of the other authors have read and approved the manuscript and no ethical issues involved.

\section{References}

Aguilar, V., C. Staver and P. Milberg, 2003. Weed vegetation response to chemical and manual selective ground cover management in a shaded coffee plantation. Weed Res., 43: 68-75. DOI: $10.1046 /$ j.1365-3180.2003.00318.x

Altieri, M.A., 1995. Agroecology: The Science of Sustainable Agriculture. 2nd Edn., Westview Press, London, ISBN-10: 1853392952, pp: 433.

Anaya, A.L., M.R. Calera, R. Mata and R. Pereda-Miranda, 1990. Allelopathic potential of compounds isolated from Ipomoea tricolor cav. (Convolvulaceae). J. Chem. Ecol., 16: 2145-2152. DOI: $10.1007 /$ BF01026926

Anonymous, 1992. Weed control in coffee. Kenya Coffee, 57: 1319-132.

Atanasov, A.G., B. Waltenberger, E.M. Pferschy-Wenzig, T. Linder and C. Wawrosch et al., 2015. Discovery and resupply of pharmacologically active plant-derived natural products: A review. Biotechnol. Adv., 33: 1582-614. DOI: 10.1016/j.biotechadv.2015.08.001

Bhadoria, P., 2011. Allelopathy: A natural way towards weed management. Am. J. Exp. Agric., 1: 7-20.

Braver, H.O., 1957. Destruccion de malas hierbas en los plantios de café. Proc. Caribb. Reg. Am. Soc. Hort. Sci., 5: 55-57.

Byrne, P.N., 1984. World Bank/Papua New Guinea Agricultural Support Services Project, Department of primary industry. Port Moresby.

Caro, P., C. Moran, B. Diaz and L. Calzado, 1990. Weed distribution in plantations, Central Cuba over two years. Café y Cacao, 12: 23-33

Cherigo, L., J. Lezcano, C. Spadafora and S. Martínez-Luis, 2012. Evaluation of phytotoxic, cytotoxic and antiparasitic in vitro activities of Borreria verticillata, a weed of Panamanian coffee crops. Biosci. Res., 9: 82-86.

Cherigo, L., M. Salazar and S. Martinez-Luis, 2015. Evaluation of phytotoxic activity of Blechum pyramidatum, a weed of Panamanian coffee crops. Int. J. Curr. Res., 7: 24172-24176.

Cragg, G.M. and D.J. Newman, 2013. Natural Products: A continuing source of novel drug leads. Biochim. Biophys. Acta, 1830: 3670-3695. DOI: $10.1016 /$ j.bbagen.2013.02.008

Dedeca, D.M., 1959. Plant pests of coffee. Sau Paulo Supt. dos Serv.do Cafe B., 34:16-20.

De Graaff, J., 1986. The Economics of Coffee. 1st Edn., Pudoc, Wageningen, ISBN-10: 9022009009, pp: 294. 
Khalid, S., T. Ahmad and R.A. Shad, 2002. Use of Allelopathy in Agriculture. Asian J. Plant Sci., 1: 292-297. DOI: 10.3923/ajps.2002.292.297

Lopez, D., L. Cherigo, C. Spadafora, M.A Loza-Mejía and S. Martínez-Luis, 2015. Phytochemical composition, antiparasitic and $\alpha$-glucosidase inhibition activities from Pelliciera rhizophorae. Chem. Cent. J., 9: 53-53. DOI: 10.1186/s13065-015-0130-3

Mata, R., M. Macias, S. Rojas, B. Lotina-Hennsen and R. Toscano et al., 1998. Phytotoxic compounds from Esenbeckia yaxhoob. Phytochemistry, 49: 441-449. DOI: 10.1016/S0031-9422(98)00110-1

Njoroge, J.M., 1994. Weeds and weed control in coffee. Expl. Agric., 30: 421-429. DOI: $10.1017 / \mathrm{S} 0014479700024662$

Ordoñez, M.A., C.J. Varela and M.H. Sosa, 2000. Manejo de Malezas en las plantaciones de café. Programa de agronomía, Honduras.
Panda, D., S. Pradhan, S.K. Palita and J.K. Nayak, 2014. Medicinal weed diversity and ethno medicinal weeds used by tribal's of Koraput, India. Eco. Env. Cons., 20: S35-S38.

Pani, D., N. Dikshit and H.N. Subudhi, 2002. Medicinal uses of rice field weeds of orissa. IJPGR, 15: 56-61.

Pino, O., Y. Sánchez and M.M. Rojas, 2013. Plant secondary metabolites as an alternative in pest management. I: Background, research approaches and trends. Rev. Protección Veg., 28: 81-94.

Radosevich, S.R., J. Holt and C. Ghersa, 1997. Weed Ecology: Implications for Management. 1st Edn., Wiley-Blackwell, USA, ISBN-10: 0471116068, pp: 608.

Ronchi, C.P. and A.A. Silva, 2006. Effects of weed species competition on the growth of young coffee plants. Planta Daninha, 4: 415-423. DOI: $10.1590 / \mathrm{S} 0100-83582006000300001$ 